\title{
The Blok-Ferreirim theorem for normal GBL-algebras and its application
}

\author{
P. Jipsen and F. Montagna
}

\begin{abstract}
Generalized basic logic algebras (GBL-algebras for short) have been introduced in [JT02] as a generalization of Hájek's BL-algebras, and constitute a bridge between algebraic logic and $\ell$-groups. In this paper we investigate normal GBL-algebras, that is, integral GBL-algebras in which every filter is normal. For these structures we prove an analogue of Blok and Ferreirim's [BF00] ordinal sum decomposition theorem. This result allows us to derive many interesting consequences, such as the decidability of the universal theory of commutative GBL-algebras, the fact that $n$-potent GBL-algebras are commutative, and a representation theorem for finite GBL-algebras as poset sums of GMV-algebras, a result which generalizes Di Nola's and Lettieri's [DL03] representation of finite BL-algebras.
\end{abstract}

\section{Introduction}

BL-algebras have been introduced by Hájek in [Ha98] as an algebraic semantics of Basic fuzzy Logic BL. This logic is a common generalization of the three most important fuzzy logics, namely Łukasiewicz, Gödel and product logics. In [CEGT00] it is shown that the variety of BL-algebras is generated by the class of residuated lattices arising from continuous t-norms on $[0,1]$ and their residuals, thus solving a problem raised in [Ha98a]. Since then, BL-algebras have been investigated in the framework of residuated lattices, cf [BT03] or [JT02] for an introduction. At the same time, the deep connections between MV-algebras, the algebraic semantics for Łukasiewicz logic, and $\ell$-groups ([Mu86], see also [Dv02] for the noncommutative case) have been extended to BL-algebras [AM03]: every totally ordered BL-algebra can be represented as an ordinal sum of an indexed family of negative cones of abelian $\ell$-groups and of MV-algebras, which in turn arise from abelian $\ell$-groups with a strong order unit via Mundici's functor $\Gamma$. (For a proof of the main result of [AM03] without using the axiom of choice see [Bu05]).

Trying to find a bridge between $\ell$-groups, which constitute a very important variety in classical algebra, and some varieties of algebras which are very important in logic, like Boolean algebras, Heyting algebras and MV-algebras, some researchers were led to a generalization of BL-algebras.

A first generalization is obtained by removing commutativity. This attempt led to the variety of pseudo BL-algebras [DGJ02], [Dv06]. Among other results, we

2000 Mathematics Subject Classification: Primary: 06F05, Secondary: 06F15, 06D35, 03 G25.

Key words and phrases: Generalized BL-algebras, residuated lattices, basic logic, generalized MV-algebras, lattice-ordered groups, hoops. 
quote a generalization, due to Dvurečenskij [Dv06], of the ordinal sum decomposition theorem in [AM03] to the noncommutative case.

Another generalization of BL-algebras is obtained by removing representability. For the $\cdot \wedge, \rightarrow, 1$ fragment, this generalization has led to the notion of hoop. In fact, these structures were introduced before BL-algebras for reasons which are independent of fuzzy logic, of [Bo69]. An interesting feature of hoops is that these structures include the $\wedge, \rightarrow, 1$ reducts of Heyting algebras. However, unlike Heyting algebras and BL-algebras, hoops need not be lattice-ordered, i.e., a pair of incomparable elements need not have a join. For hoops, an important representation theorem is the Blok-Ferreirim theorem [BF00], which states that a subdirectly irreducible hoop is representable as an ordinal sum of a proper subhoop of it and a Wajsberg hoop, which is either a negative cone of an abelian $\ell$-group, or the $\cdot, \rightarrow, 1$ reduct of an MV-algebra. A noncommutative variant of hoops, called pseudo hoops, has also been investigated [GLP05].

But in our opinion, the most elegant generalization of BL-algebras is constituted by the GBL-algebras, see [JT02] and [GT05]. These algebras are a common generalization of $\ell$-groups and of Heyting algebras, and therefore they constitute a bridge between algebra and substructural logics. The most important properties which are shared by $\ell$-groups and Heyting algebras, and which are the defining properties of GBL-algebras, are: (a) they are both residuated lattices (for $\ell$-groups, residuals are given by $x / y=x y^{-1}$ and $y \backslash x=y^{-1} x$ ); (b) they both enjoy the divisibility property: if $x \leq y$, then there are $z, u$ such that $y z=u y=x$ (for $\ell$-groups it suffices to take $z=y^{-1} x$ and $u=x y^{-1}$, for Heyting algebras, take $\left.u=z=y \rightarrow x\right)$. Note that some properties, like the identity $x(x \backslash e)=e$ or cancellativity, are satisfied by $\ell$-groups but not by Heyting algebras; other properties, like integrality $(x \leq e)$, commutativity, idempotence $\left(x^{2}=x\right)$ and boundedness (existence of a minimum and of a maximum element) are satisfied by Heyting algebras and not by $\ell$-groups.

Whilst the literature on BL-algebras and on pseudo BL-algebras is rapidly increasing, at this moment only a few results, although significant, are known about GBL-algebras. The deepest result in this field is a theorem by Galatos and Tsinakis, saying that every GBL-algebra decomposes as a direct product of an $\ell$-group and an integral GBL-algebra, [GT05]. Another interesting result [JM06] states that every finite GBL-algebra is commutative and integral.

In this paper we continue the investigation of GBL-algebras, focusing on the representation theorems. First of all, we extend the Blok-Ferreirim theorem [BF00] to normal GBL-algebras (a GBL-algebra is said to be normal iff every filter of it is a normal filter). A consequence of the Blok-Ferreirim decomposition for normal GBL-algebras is a generalization of [JM06]: every $n$-potent GBL-algebra (i.e., every GBL-algebra satisfying the equation $x^{n+1}=x^{n}$ for some $n$ ) is commutative. As another application we prove that the variety of commutative and integral GBLalgebras has the finite embeddability property. It follows that the universal theory of commutative GBL-algebras is decidable. The final section of the paper introduces poset sums for residuated lattices as a generalization of direct products and ordinal 
sums. This concept and the Blok-Ferreirim decomposition are then used to prove that every finite GBL-algebra is represented as the poset sum of finite Wajsberg chains. As a consequence one obtains a bijective correspondence between finite GBL-algebras and finite posets labeled by natural numbers.

\section{Basic notions}

Definition 1. A residuated lattice [JT02] is a system $\mathbf{L}=(L, \cdot, \backslash, /, \vee, \wedge, e)$ where

(a) $(L, \cdot, e)$ is a monoid,

(b) $(L, \vee, \wedge)$ is a lattice,

(c) \and / are binary operations such that the residuation property holds: $x \cdot y \leq z$ iff $y \leq x \backslash z$ iff $x \leq z / y$.

In the sequel the symbol $\cdot$ will be often omitted.

Definition 2. A residuated lattice is:

- commutative if it satisfies $x y=y x$;

- integral if it satisfies $x \leq e$;

- divisible iff $x \leq y$ implies $y(y \backslash x)=(x / y) y=x$;

- cancellative if $x y w=x z w$ implies $y=z$;

- representable if it is isomorphic to a subdirect product of totally ordered residuated lattices;

- bounded if it has a minimum element $m$ and the language has an additional constant 0 which is interpreted as $m$.

Note that a bounded residuated lattice also has a maximum, namely $0 / 0$. Moreover, all of the above properties can be expressed by equations, cf [BT03], [JT02] and [GO06]. Note also that $\ell$-groups (i.e., groups with a multiplication that distributes over the lattice operations) can be presented as residuated lattices satisfying $x(x \backslash e)=e$. Indeed, given an $\ell$-group we obtain a cancellative and divisible residuated lattice letting $x \backslash y=x^{-1} y$ and $y / x=y x^{-1}$. Conversely, from a residuated lattice satisfying $x(x \backslash e)=e$ we obtain an $\ell$-group letting $x^{-1}=x \backslash e=e / x$.

In a commutative residuated lattice the operations $x \backslash y$ and $y / x$ coincide and are denoted by $x \rightarrow y$.

Definition 3. A GBL-algebra (cf [JT02], [GT05]) is a divisible residuated lattice. A $G M V$-algebra is a GBL-algebra satisfying the equation $y /((x \backslash y) \wedge e))=$ $((y / x) \wedge e) \backslash y=x \vee y$. A BL-algebra [Ha98] is a commutative, integral, bounded and representable GBL-algebra. An $M V$-algebra [COM00] is a commutative and bounded GMV-algebra. (Note that commutative GMV-algebras, and hence MValgebras, are always representable, see e.g. [GT05].)

We note that bounded integral GMV-algebras are usually called pseudo- $M V$ algebras, but have also been called generalized $M V$-algebras or $G M V$-algebras. To avoid confusion, we only use the name "GMV-algebras" in its most general sense.

In any residuated lattice, the following conditions hold (cf [JT02], [GT05]). 
(1) $x(x \backslash y) \leq y$ and $(y / x) x \leq y$

(2) $x(y \vee z)=(x y) \vee(y z)$ and $(y \vee z) x=(y x) \vee(z x)$

(3) $(x \vee y) \backslash z=(x \backslash z) \wedge(y \backslash z)$ and $z /(x \vee y)=(z / x) \wedge(z / y)$

(4) $z \backslash(x \wedge y)=(z \backslash x) \wedge(z \backslash y)$ and $(x \wedge y) / z=(x / z) \wedge(y / z)$

(5) $(x / y) / z=x /(z y)$ and $z \backslash(y \backslash x)=(y z) \backslash x$

(6) $x \backslash(z / y)=(x \backslash z) / y$

(7) $x \leq y /(x \backslash y)$ and $x \leq(y / x) \backslash y$

(8) $(x \backslash y)=(y /(x \backslash y)) \backslash y$ and $(y / x)=y /((y / x) \backslash y)$.

Moreover, any GBL-algebra has a distributive lattice reduct, and any integral GBL-algebra satisfies:

(9) $(y / x) \backslash(x / y)=x / y$ and $(y \backslash x) /(x \backslash y)=y \backslash x$.

(10) $(x / y) y=y(y \backslash x)=x \wedge y$.

In [GT05], the following result is shown.

Proposition 4. Every GBL-algebra is isomorphic to the direct product of an $\ell$ group and an integral GBL-algebra.

Thus in the sequel we will mainly concentrate on integral GBL-algebras.

Lemma 5. An integral GBL-algebra $\mathbf{A}$ is a $G M V$-algebra iff for all $a, b \in A$ one has: $(a \vee b) \backslash a=a$ iff $a \vee b=e$ iff $a /(a \vee b)=a$.

Proof. In any integral GMV-algebra we have $a /((a \vee b) \backslash a)=a \vee b=(a /(a \vee b)) \backslash a$ and $e \backslash a=a / e=a$, therefore $(a \vee b) \backslash a=a$ iff $a \vee b=e$ iff $a /(a \vee b)=a$. Conversely, assume that $(a \vee b) \backslash a=a$ iff $a \vee b=e$ iff $a /(a \vee b)=a$ for all $a, b \in A$. We show that for all $x, y \in A$ we have $x /((x \vee y) \backslash x)=(x /(x \vee y)) \backslash x=x \vee y$. Since $(x \vee y) \backslash x=y \backslash x$ and $x /(x \vee y)=x / y$ holds in any integral residuated lattice, this will imply the characteristic equation of integral GMV-algebras, namely, $x /(y \backslash x)=$ $(x / y) \backslash x=x \vee y$. Now let $u=x /((x \vee y) \backslash x)$. Then by (7) and (8) we have:

(11) $x \vee y \leq u$ and $u \backslash x=(x \vee y) \backslash x$.

Thus using (11), (10) and (5) we get

$$
\begin{aligned}
(x \vee y) \backslash x & =(u \wedge(x \vee y)) \backslash x=(u(u \backslash(x \vee y))) \backslash x=(u \backslash(x \vee y)) \backslash(u \backslash x)= \\
& =(u \backslash(x \vee y)) \backslash((x \vee y) \backslash x) .
\end{aligned}
$$

Therefore, letting $a=(x \vee y) \backslash x$ and $b=u \backslash(x \vee y)$, we have $a=b \backslash a$. But $b=$ $u \backslash(x \vee y) \geq u \backslash x=(x \vee y) \backslash x=a$, whence $b=a \vee b$, and $a=(a \vee b) \backslash a$. It follows that $b=a \vee b=e$. Hence, $u \leq x \vee y$, and finally, by (11), $u=x \vee y$. The proof of $(x /(x \vee y)) \backslash x=x \vee y$ is symmetric.

Definition 6. A hoop (cf [Bo69] [BO75] [BF00]) is a commutative and integral residuated partially ordered monoid $(M, \cdot, \rightarrow, e)$, with partial order $\leq$ defined by $x \leq y$ iff $x \rightarrow y=e$, satisfying the divisibility condition: $x \leq y$ iff $y \cdot(y \rightarrow x)=x$. A hoop is said to be a Wajsberg hoop iff it is a subreduct of an MV-algebra. A Wajsberg chain is a totally ordered Wajsberg hoop. 
It can be proved that hoops are precisely the subreducts of commutative and integral GBL-algebras with respect to the signature $\{\cdot, \rightarrow, e\}$. Moreover in any hoop the meet operation is definable by $x \wedge y=x \cdot(x \rightarrow y)$, and in a Wajsberg hoop, the join is also definable by $x \vee y=(x \rightarrow y) \rightarrow y$, cf [BF00], [Fe92]. Thus Wajsberg hoops are term-equivalent to commutative and integral GMV-algebras.

Definition 7. The ordinal sum $\mathbf{H}_{1} \oplus \mathbf{H}_{2}$ (cf [Co82], [BF00]) of two hoops $\mathbf{H}_{1}$ and $\mathbf{H}_{2}$ is defined as follows: up to isomorphism, we may assume that $H_{1} \cap H_{2}=$ $\left\{e_{H_{1}}\right\}=\left\{e_{H_{2}}\right\}$ (in the sequel we denote $e_{H_{1}}=e_{H_{2}}$ by $e$ ). The universe of $\mathbf{H}_{1} \oplus \mathbf{H}_{2}$ is $H_{1} \cup H_{2}$, and the top element is $e$. The operations are defined as follows:

$$
\begin{array}{r}
x \cdot y= \begin{cases}x \cdot_{i} y & \text { if } x, y \in H_{i}(i=1,2) \\
x & \text { if } x \in H_{1} \backslash\{e\}, y \in H_{2} \\
y & \text { if } y \in H_{1} \backslash\{e\}, x \in H_{2}\end{cases} \\
x \rightarrow y= \begin{cases}x \rightarrow_{i} y & \text { if } x, y \in H_{i}(i=1,2) \\
e & \text { if } x \in H_{1} \backslash\{e\}, y \in H_{2} \\
y & \text { if } y \in H_{1} \backslash\{e\}, x \in H_{2}\end{cases}
\end{array}
$$

Building on work of Büchi and Owens [BO75], Blok and Ferreirim [BF00] proved the following result.

Proposition 8. Every subdirectly irreducible hoop is the ordinal sum of a proper subhoop $\mathbf{H}$ and a subdirectly irreducible nontrivial Wajsberg hoop $\mathbf{W}$.

We are going to extend the notion of ordinal sum to integral GBL-algebras. If we just copy the definition of ordinal sum of hoops, we meet a difficulty: if $e$ is not join irreducible in $\mathbf{H}_{1}$, and $\mathbf{H}_{2}$ has no minimum, then the ordinal sum defined as for hoops is not closed under join. Indeed, let $a, b<e$ in $\mathbf{H}_{1}$ such that $a \vee_{1} b=e$. Then $a \vee b$ does not exist in $\mathbf{H}_{1} \oplus \mathbf{H}_{2}$, because the upper bounds of $a, b$ are precisely the elements of $\mathbf{H}_{2}$, and this set has no minimum. Thus the ordinal sum construction splits into the following cases:

Ordinal sums of type (a). If $e$ is join irreducible in $\mathbf{H}_{1}$, then we can define the ordinal sum of $\mathbf{H}_{1}$ and $\mathbf{H}_{2}$ in a way very similar to the case of hoops. Of course we have to take into consideration that we now have two different residuals, and, more importantly, we also have to define join and meet. Multiplication and residuals are defined in the obvious way:

$$
\begin{aligned}
& x \cdot y=\left\{\begin{array}{lll}
x \cdot_{i} y & \text { if } & x, y \in H_{i}(i=1,2) \\
x & \text { if } & x \in H_{1} \backslash\{e\}, y \in H_{2} \\
y & \text { if } & y \in H_{1} \backslash\{e\}, x \in H_{2}
\end{array}\right. \\
& x \backslash y=\left\{\begin{array}{lll}
x \backslash_{i} y & \text { if } & x, y \in H_{i}(i=1,2) \\
e & \text { if } & x \in H_{1} \backslash\{e\}, y \in H_{2} \\
y & \text { if } & y \in H_{1} \backslash\{e\}, x \in H_{2}
\end{array}\right. \\
& y / x=\left\{\begin{array}{lll}
y /{ }_{i} x & \text { if } & x, y \in H_{i}(i=1,2) \\
e & \text { if } & x \in H_{1} \backslash\{e\}, y \in H_{2} \\
y & \text { if } & y \in H_{1} \backslash\{e\}, x \in H_{2}
\end{array}\right.
\end{aligned}
$$


Meet is definable as $x \wedge y=x(x \backslash y)$. In this way, we have:

$$
x \wedge y=\left\{\begin{array}{lll}
x \wedge_{i} y & \text { if } & x, y \in H_{i}(i=1,2) \\
x & \text { if } & x \in H_{1} \backslash\{e\}, y \in H_{2} \\
y & \text { if } & y \in H_{1} \backslash\{e\}, x \in H_{2}
\end{array}\right.
$$

Moreover, it is easily seen that $\mathbf{H}_{1} \oplus \mathbf{H}_{2}$ is closed under join and that the join operation is defined by:

$$
x \vee y=\left\{\begin{array}{lll}
x \vee_{i} y & \text { if } & x, y \in H_{i}(i=1,2) \\
x & \text { if } & y \in H_{1} \backslash\{e\}, x \in H_{2} \\
y & \text { if } & x \in H_{1} \backslash\{e\}, y \in H_{2}
\end{array}\right.
$$

Ordinal sums of type (b). If $e$ is not join-irreducible in $\mathbf{H}_{1}$ and $\mathbf{H}_{2}$ has a minimum $m$ then $\mathbf{H}_{1} \oplus \mathbf{H}_{2}$ is defined as in case (a) with the exception of join. Indeed, if $x \vee_{1} y=e$ in $\mathbf{H}_{1}$, then the least upper bound of $x$ and $y$ in $\mathbf{H}_{1} \oplus \mathbf{H}_{2}$ is the minimum of $\mathbf{H}_{2}$. It follows that join in $\mathbf{H}_{1} \oplus \mathbf{H}_{2}$ is defined as follows:

$$
x \vee y= \begin{cases}x \vee_{2} y & \text { if } \quad x, y \in H_{2} \\ x \vee_{1} y & \text { if } x, y \in H_{1} \text { and } x \vee_{1} y<e \\ m & \text { if } x, y \in H_{1} \backslash\{e\} \text { and } x \vee_{1} y=e \\ x & \text { if } y \in H_{1} \backslash\{e\}, x \in H_{2} \\ y & \text { if } x \in H_{1} \backslash\{e\}, y \in H_{2}\end{cases}
$$

Ordinal sums of type (c). If $e$ is not join irreducible in $\mathbf{H}_{1}$, and $\mathbf{H}_{2}$ has no minimum, then the ordinal sum $\mathbf{H}_{1} \oplus \mathbf{H}_{2}$ defined as in the case of hoops is not closed under join. Therefore we have to change the whole construction slightly: replacing, if necessary, $\mathbf{H}_{1}$ and $\mathbf{H}_{2}$ by isomorphic copies, we can assume without loss of generality that $H_{1} \cap H_{2}=\emptyset$. Then define $\mathbf{H}_{1} \oplus \mathbf{H}_{2}$ as follows:

The universe of $\mathbf{H}_{1} \oplus \mathbf{H}_{2}$ is $H_{1} \cup H_{2}$, and $e_{H_{2}}$ is both the top element and the monoid unit. The operations are as follows:

$$
\begin{aligned}
& x \cdot y=\left\{\begin{array}{lll}
x \cdot{ }_{i} y & \text { if } & x, y \in H_{i}(i=1,2) \\
x & \text { if } \quad x \in H_{1}, y \in H_{2} \\
y & \text { if } \quad y \in H_{1}, x \in H_{2}
\end{array}\right.
\end{aligned}
$$

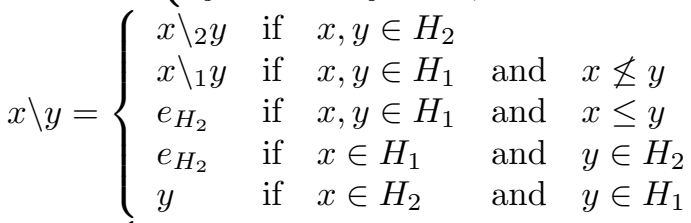

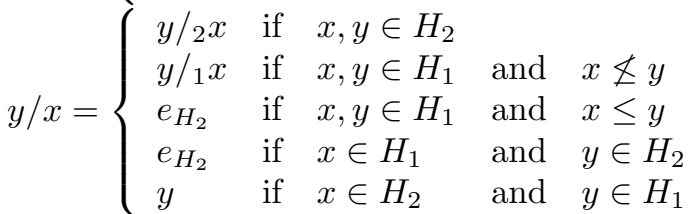

$$
\begin{aligned}
& x \wedge y= \begin{cases}x \wedge_{i} y & \text { if } \quad x, y \in H_{i}(i=1,2) \\
x & \text { if } \quad x \in H_{1}, y \in H_{2} \\
y & \text { if } \quad y \in H_{1}, x \in H_{2}\end{cases}
\end{aligned}
$$




$$
x \vee y=\left\{\begin{array}{lll}
x \vee_{i} y & \text { if } & x, y \in H_{i}(i=1,2) \\
x & \text { if } & y \in H_{1}, x \in H_{2} \\
y & \text { if } & x \in H_{1}, y \in H_{2}
\end{array}\right.
$$

It is readily seen that in all cases $\mathbf{H}_{1} \oplus \mathbf{H}_{2}$ is an integral GBL-algebra if $\mathbf{H}_{1}$ and $\mathbf{H}_{2}$ are (verification is left to the reader). We note that ordinal sums as defined here can also be applied to integral residuated lattices in general.

In an ordinal sum $\mathbf{H}_{1} \oplus \mathbf{H}_{2}$ of any of the types (a), (b) or (c), of two integral GBL-algebras, $\mathbf{H}_{2}$ is always a subalgebra of $\mathbf{H}_{1} \oplus \mathbf{H}_{2}$. In ordinal sums of type (a), $\mathbf{H}_{1}$ is also a subalgebra of $\mathbf{H}_{1} \oplus \mathbf{H}_{2}$. In ordinal sums of type (b), $\mathbf{H}_{1}$ is closed under all operations except from join, whereas in ordinal sums of type (c), $\mathbf{H}_{1}$ is not even closed under residuals.

Ordinal sums of type (a) can be generalized in an obvious way to the case of infinitely many summands. In this case we consider a totally ordered set $I$ of indices, and for all $i \in I$ we consider an integral GBL-algebra $\mathbf{H}_{i}$ in such a way that for $i \neq j, \mathbf{H}_{i} \cap \mathbf{H}_{j}=\left\{e_{i}\right\}=\left\{e_{j}\right\}=\{e\}$, and for all $i, e_{i}$ is join irreducible in $\mathbf{H}_{i}$. Then the ordinal sum $\bigoplus_{i \in I} \mathbf{H}_{i}$ is defined as follows:

- The universe of $\bigoplus_{i \in I} \mathbf{H}_{i}$ is $\bigcup_{i \in I} H_{i}$.

- $x \cdot y=\left\{\begin{array}{lll}x \cdot{ }_{i} y & \text { if } & x, y \in H_{i}(i \in I) \\ x & \text { if } & x \in H_{i} \backslash\{e\}, y \in H_{j} \text { with } i<j \\ y & \text { if } & y \in H_{i} \backslash\{e\}, x \in H_{j} \text { with } i<j\end{array}\right.$

- The partial order on $\bigoplus_{i \in I} \mathbf{H}_{i}$ is the unique partial order $\leq$ such that: (i) $e$ is the top element with respect to $\leq_{\text {; }}$ (ii) the partial order $\leq_{i}$ on $\mathbf{H}_{i}$ is the restriction of $\leq$ to $\mathbf{H}_{i}$, and (iii) if $i<j$, then every element of $H_{i} \backslash\{e\}$ precedes every element of $H_{j}$.

- The lattice operations and the residuals are uniquely determined by $\leq$ and by $\cdot$.

In [AM03] the following is shown.

Proposition 9. Every totally ordered commutative and integral GBL-algebra $\mathbf{A}$ can be represented as $\bigoplus_{i \in I} \mathbf{H}_{i}$, where for all $i \in I, \mathbf{H}_{i}$ is a commutative, integral and totally ordered $G M V$-algebra. If in addition $\mathbf{A}$ is a BL-algebra, then I must have a minimum $i_{0}$ and $\mathbf{H}_{i_{0}}$ must be an $M V$-algebra.

Recently Dvurečenskij [Dv06] has shown that Proposition 9 extends to the noncommutative case.

\section{The Blok-Ferreirim decomposition theorem for integral normal GBL- algebras}

Proposition 8 does not extend to integral GBL-algebras. In [JM06] the authors present an example of a subdirectly irreducible integral GBL-algebra which cannot be decomposed as an ordinal sum of two GBL-algebras and is not a GMV-algebra. In this section we find some classes of integral GBL-algebras which satisfy the Blok-Ferreirim decomposition theorem. 
Definition 10. A filter of a residuated lattice $\mathbf{A}$ is a set $F \subseteq A$ such that $e \in F$, $F$ is upward closed and $F$ is closed under product and under meet. A filter is said to be normal iff $a \in F$ implies $b \backslash(a b) \in F$ and $(b a) / b \in F$ for every $b \in A$.

Note that in an integral residuated lattice, normal filters are precisely the congruence classes of $e$. In the nonintegral case, normal filters are the lattice filters generated by the congruence classes of $e$. Since residuated lattices are $e$-regular, it follows that normal filters are in one-to-one correspondence with congruences via the map $F \mapsto\{(a, b): a / b, b / a \in F\}$ (see [BT03], [JT02]).

Definition 11. Let $\mathbf{L}$ be a residuated lattice and let $a \in L$ with $a \leq e$. We say that $a$ is stable (cf [GOR]) iff for all $c \in L$ there is a natural number $n$ such that $a^{n} c \leq c a$ and $c a^{n} \leq a c$. We say that $\mathbf{L}$ is:

- weakly commutative if for every $a \in L, a \wedge e$ is stable,

- normal if every filter of it is normal, and

- $n$-potent if it satisfies $x^{n+1}=x^{n}$.

The following result is a particular case of [GOR], Corollary 10.

Proposition 12. In any residuated lattice $\mathbf{L}$, the following are equivalent:

(a) Every element $a \leq e$ is stable.

(b) For all $b \in L$, the normal filter $\mathrm{Fg}(b)$ generated by $b$ is given by the formula $\mathrm{Fg}(b)=\left\{x \in L: \exists n \in \omega\left((b \wedge e)^{n} \leq x\right)\right\}$.

As an immediate consequence, we have the following result.

Corollary 13. An integral residuated lattice is weakly commutative iff it is normal.

Lemma 14. Let $\mathbf{L}$ be an integral and normal residuated lattice and let $x, y \in L$. Then the following hold.

(a) There is $n \in \omega$ such that $x \backslash y \leq y / x^{n}$ and $y / x \leq x^{n} \backslash y$.

(b) $x \backslash y=y$ iff for all $n \in \omega, x^{n} \backslash y=y$ iff for all $n \in \omega, y / x^{n}=y$ iff $y / x=y$.

Proof. (a) By Corollary 13, $\mathbf{L}$ is weakly commutative, therefore for all $x, y \in L$ there is $n \in \omega$ such that $(x \backslash y) x^{n} \leq x(x \backslash y) \leq y$ and $x^{n}(y / x) \leq(y / x) x \leq y$. By residuation, it follows that $x \backslash y \leq y / x^{n}$ and $y / x \leq x^{n} \backslash y$.

(b) Suppose $x \backslash y=y$. Then $x^{2} \backslash y=x \backslash(x \backslash y)=x \backslash y=y$, and iterating we get $x^{n} \backslash y=y$ for every $n$. Now by (a) there is $n$ such that $y \leq y / x \leq x^{n} \backslash y=y$, therefore $y / x=y$. By a symmetric argument we get that $y / x=y$ implies $y / x^{n}=y$ for every $n$ and this in turn implies $x \backslash y=y$, hence the claim is proved.

Lemma 15. Every n-potent GBL-algebra $\mathbf{A}$ is integral and normal.

Proof. By Proposition 4, A decomposes as a direct product of an integral GBLalgebra and an $\ell$-group. Since any $n$-potent $\ell$-group is trivial, $\mathbf{A}$ is integral. With regards to normality, for all $x \in A, x^{n}$ is an idempotent, and by [JM06], $x^{n} y=$ $y x^{n}=x^{n} \wedge y$. Thus we get $x^{n} y=y x^{n} \leq y x$ and $y x^{n}=x^{n} y \leq x y$. It follows that A is weakly commutative, therefore by Corollary 13 it is normal. 
Lemma 15 holds for $n$-potent GBL-algebras but not for $n$-potent residuated lattices in general. Indeed, in [GOR] the authors present an example of a finite and integral (hence $n$-potent for some $n$ ) residuated lattice which is not normal.

Clearly, commutative GBL-algebras are normal, but the converse does not hold, as illustrated by the next example.

Example 16. An $\ell$-group is weakly abelian if it satisfies $x^{2} y \leq y x$ for each $x \leq e$ and for all $y$. Note that there are weakly abelian and nonabelian $\ell$-groups [AF88]. Note also that a weakly abelian $\ell$-group satisfies $y x^{2} \leq x y$ whenever $x \leq e$ : replacing $y$ by $y^{-1}$ in the definition of weakly abelian $\ell$-group and multiplying both sides by $y$ to the left and to the right, we get $y\left(x^{2} y^{-1}\right) y=y x^{2} \leq y\left(y^{-1} x\right) y=x y$. Thus any negative cone of a weakly abelian, but not abelian, $\ell$-group with residuals defined by $x \backslash y=\left(x^{-1} y\right) \wedge e$ and $y / x=\left(y x^{-1}\right) \wedge e$, is a weakly commutative but not commutative integral GBL-algebra. The same is true of the ordinal sum of two (or more) negative cones of weakly abelian $\ell$-groups.

Let $\mathbf{A}$ be a subdirectly irreducible normal and integral GBL-algebra and let $M$ be its minimum nontrivial filter. An element $a \in A$ is said to be fixed if $a<e$ and there exists $m \in M \backslash\{e\}$ such that $m \backslash a=a / m=a$. In the sequel, $F$ will denote the set of fixed elements of $\mathbf{A}$ and $F^{+}$will denote the set $F \cup\{e\}$. Finally, we define $M^{-}=M \backslash\{e\}$ and $S=A \backslash F$. We establish a sequence of lemmas in full analogy with [BF00].

Lemma 17. (a) For every $a \in A \backslash\{e\}$ there is $m \in M^{-}$such that $a \leq m$.

(b) For $m, m^{\prime} \in M,\left(m \vee m^{\prime}\right) \backslash m^{\prime}=m^{\prime}$ implies $m \vee m^{\prime}=e$ and $m^{\prime} /\left(m \vee m^{\prime}\right)=$ $m^{\prime}$ implies $m \vee m^{\prime}=e$. Thus, (by Lemma 5), $M$ is the domain of an integral GMV-algebra.

(c) If $f \in F$, then for all $m \in M$ we have $f / m=m \backslash f=f$.

(d) $M \cap F=\emptyset$.

(e) For $f \in F$ and $m \in M$, one has $f \leq m$.

Proof. (a) Let $m^{\prime} \in M^{-}$. By the minimality of $M, m^{\prime}$ belongs to the normal filter generated by $a$, and by the normality and the integrality of $\mathbf{A}$, there exists a positive $n$ such that $a^{n} \leq m^{\prime}$. Let $n$ be the least positive integer with this property. Then $a^{n-1} \backslash m^{\prime}<e$ and $a \leq a^{n-1} \backslash m^{\prime}$. Finally, $m^{\prime} \leq a^{n-1} \backslash m^{\prime}$, therefore $a^{n-1} \backslash m^{\prime} \in M^{-}$. Thus $m=a^{n-1} \backslash m^{\prime}$ meets our requirements.

(b) Suppose $\left(m \vee m^{\prime}\right) \backslash m^{\prime}=m^{\prime}$. If $m \vee m^{\prime}<e$, then the principal filter generated by $m \vee m^{\prime}$ is the minimum nontrivial normal filter, therefore there exists $k$ such that $\left(m \vee m^{\prime}\right)^{k} \leq m^{\prime}$. Moreover by Lemma 14 (b), we have $\left(m \vee m^{\prime}\right)^{k} \backslash m^{\prime}=m^{\prime}<e$, and a contradiction has been reached. Again by Lemma 14 (b), $m^{\prime} /\left(m \vee m^{\prime}\right)=m^{\prime}$ implies $\left(m \vee m^{\prime}\right) \backslash m^{\prime}=m^{\prime}$, which in turn implies $m \vee m^{\prime}=e$. Now every filter of an integral GBL-algebra is a subalgebra of it. Thus $M$ is the domain of an integral GBL-algebra, which is a GMV-algebra by Lemma 5 .

(c) Let $f \in F$ and let $m \in M^{-}$be such that $f / m=m \backslash f=f$. Let $m^{\prime} \in M$. Then there exists $n \in \omega$ such that $m^{n} \leq m^{\prime}$, hence $m^{\prime} \backslash f \leq m^{n} \backslash f=f$, therefore $m^{\prime} \backslash f=f$. By Lemma 14 (b), $f / m^{\prime}=f$. 
(d) By (c), if $m \in M \cap F$, then $m \backslash m=m$, therefore $m=e$, a contradiction.

(e) By (c), one has $f / m=m \backslash f=f$. Moreover by the formula (9) in Section 2 , we have $(f / m) \backslash(m / f)=m / f$, therefore letting $x=f / m=f$ and $y=m / f$, we have $x \backslash y=y$, which by Lemma 14 (b), implies $y=x^{n} \backslash y$ for every $n$. Now $y=m / f \geq m \in M$, therefore for some $n, x^{n} \leq y$, because the filter generated by $x$ contains $M$. This implies $y=e$ and finally $f \leq m$.

Lemma 18. Let $m \in M, s \in S$ and $f \in F$. Then

(a) $m f=f m=f$,

(b) $f^{\prime} \leq f$ implies $f^{\prime} \in F$,

(c) $F^{+}=F \cup\{e\}$ is closed under all the operations of residuated lattices, with the possible exception of $\vee$,

(d) $f \leq s$, fs $=s f=f \wedge s$ and $s \backslash f=f / s=f$,

(f) $S$ is the domain of a subalgebra of $\mathbf{A}$,

(g) $S$ is the domain of an integral GMV-algebra.

Proof. (a) By Lemma 17 (e), we have $f \leq m$, therefore $f=f \wedge m=m(m \backslash f)=$ $(f / m) m=m f=f m$.

(b) We have $\left(m \backslash f^{\prime}\right) \leq m \backslash f=f$, therefore $m \backslash f^{\prime}=\left(m \backslash f^{\prime}\right) \wedge f$. Now let us compute (using repeatedly $x(x \backslash y)=x \wedge y$ and $x \backslash(y \backslash z)=(y x) \backslash z)$

$$
\begin{aligned}
\left(m \backslash f^{\prime}\right) \backslash f^{\prime} & =\left(\left(m \backslash f^{\prime}\right) \wedge f\right) \backslash f^{\prime} \\
& =\left(f\left(f \backslash\left(m \backslash f^{\prime}\right)\right) \backslash f^{\prime}\right. \\
& =\left(f \backslash\left(m \backslash f^{\prime}\right)\right) \backslash\left(f \backslash f^{\prime}\right) \\
& =\left((m f) \backslash f^{\prime}\right) \backslash\left(f \backslash f^{\prime}\right) \\
& =\left(f \backslash f^{\prime}\right) \backslash\left(f \backslash f^{\prime}\right) \\
& =e
\end{aligned}
$$

(recall that by (a), $m f=f$ ). Thus $m \backslash f^{\prime} \leq f^{\prime}$, and $m \backslash f^{\prime}=f^{\prime}$. By Lemma 14 (b), $f^{\prime} / m=f^{\prime}$, therefore $f^{\prime} \in F$.

(c) Let $f_{1}, f_{2} \in F$. Then $m \backslash\left(f_{1} \backslash f_{2}\right)=\left(f_{1} m\right) \backslash f_{2}=f_{1} \backslash f_{2}$, and by Lemma 14 (b), $\left(f_{1} \backslash f_{2}\right) / m=f_{1} \backslash f_{2}$. Thus either $f_{1} \backslash f_{2}=e$ or $f_{1} \backslash f_{2} \in F$. It follows that $F^{+}$is closed under $\backslash$ and $/$. That $F^{+}$is closed under product and under meet is clear, as $F$ is downward closed.

(d) We have $m \backslash(f \backslash s)=(f m) \backslash s=f \backslash s$. By Lemma 14 (b), $(f \backslash s) / m=f \backslash s$. Thus either $f \backslash s \in F$ or $f \backslash s=e$. But since $s \leq f \backslash s$ and $F$ is downward closed, $f \backslash s \in F$ would imply $s \in F$, which is impossible. Thus $f \backslash s=e$ and $f \leq s$.

Now $((f / s) \backslash f) / m=(f / s) \backslash(f / m)=(f / s) \backslash f$. Therefore by Lemma 14 (b), $m \backslash((f / s) \backslash f)=(f / s) \backslash f$ and $(f / s) \backslash f$ is either $e$ or in $F$. Note that $S$ is upward closed, since $F$ is downward closed. Thus since $s \leq(f / s) \backslash s$, we have $(f / s) \backslash f \in S$ and $(f / s) \backslash f \notin F$. Hence $(f / s) \backslash f=e$, therefore $f / s \leq f$ and $f / s=f$. By Lemma 14 (b), $s \backslash f=f$.

Finally $f=f \wedge s=s(s \backslash f)=(f / s) s=s f=f s$.

(e) Since $S$ is upward closed, it is closed under $\backslash$, under / and under join. We prove that $S$ is closed under product. Suppose $s, s^{\prime} \in S$. Without loss of generality, 
we may assume $s, s^{\prime}<e$. Then for all $f \in F,\left(s s^{\prime}\right) \backslash f=s^{\prime} \backslash(s \backslash f)=s^{\prime} \backslash f=f$. By Lemma 14 (b), $f /\left(s s^{\prime}\right)=f$. Thus $s s^{\prime} \notin F$, otherwise we would get $e=\left(s s^{\prime}\right) \backslash\left(s s^{\prime}\right)=$ $s s^{\prime}$. It follows that $s s^{\prime} \in S$. Finally $s \wedge s^{\prime} \in S$, because $s \wedge s^{\prime} \geq s s^{\prime}$, and $S$ is upward closed.

(f) Suppose $\left(s \vee s^{\prime}\right) \backslash s=s$ with $s, s^{\prime} \in S$. If $s \vee s^{\prime}<e$, then by Lemma 17, (a) there is an element $m \in M^{-}$such that $s \vee s^{\prime} \leq m$. But then $s \leq m \backslash s \leq\left(s \vee s^{\prime}\right) \backslash s=s$, and by Lemma 14 (b), $m \backslash s=s / m=s$. This implies $s \in F$, a contradiction. It follows that $\left(s \vee s^{\prime}\right) \backslash s=s$ implies $s \vee s^{\prime}=e$. By Lemma 14 (b), $s /\left(s \vee s^{\prime}\right)=s$ implies $\left(s \vee s^{\prime}\right) \backslash s=s$ and $s \vee s^{\prime}=e$. Thus by Lemma $5, S$ is the domain of a GMV-algebra.

We have seen that $S$ is the domain of a subalgebra, $\mathbf{S}$, of $\mathbf{A}$ which is an integral GMV-algebra. Moreover $F^{+}=F \cup\{e\}$ is closed under all operations of residuated lattices except possibly join. If $F$ is also closed under join, then $F^{+}$is the domain of a subalgebra, $\mathbf{F}$, of $\mathbf{A}$ and $\mathbf{A}$ is the ordinal sum of type (a) of $\mathbf{F}$ and $\mathbf{S}$. Otherwise, note that every element of $S$ is an upper bound of $F$, therefore if two elements of $F$ have no upper bound in $F$, then their join must be the minimum of $\mathbf{S}$ (recall that $\mathbf{A}$ is closed under join). In this case, define for $a, b \in F^{+}, a \vee_{F} b=a \vee b$ if $a, b \in F$ and the join of $a$ and $b$ exists in $F$, and $a \vee_{F} b=e$ otherwise. Let us denote by $\mathbf{F}$ the resulting algebra. Then $\mathbf{A}$ is the ordinal sum of type (b) of $\mathbf{F}$ and $\mathbf{S}$. Summing up, we have:

Theorem 19. Every normal subdirectly irreducible integral GBL-algebra decomposes as the ordinal sum, either of type (a) or of type (b), of an integral GBL-algebra and a subdirectly irreducible integral nontrivial GMV-algebra.

\section{4. n-potent GBL-algebras are commutative}

In [JM06] it is shown that every finite GBL-algebra is commutative. In this section we extend the result to $n$-potent GBL-algebras. Every finite GBL-algebra is integral and hence $n$-potent for some $n$, but the converse does not hold. Therefore the result we present here is a strengthening of [JM06]. We begin by recalling the following observation from that paper.

Lemma 20. Let $\mathbf{A}$ be any integral GBL-algebra.

(a) If $x$ is an idempotent element of $\mathbf{A}$, then for all $y \in A, x y=y x=x \wedge y$.

(b) The set of idempotent elements of $\mathbf{A}$ is closed under join and meet.

Proof. (a) $x y \leq x \wedge y=x(x \backslash y)=x^{2}(x \backslash y)=x(x \wedge y) \leq x y$.

(b) If $x^{2}=x$ and $y^{2}=y$, then $(x \vee y)^{2}=x^{2} \vee x y \vee y x \vee y^{2}=x \vee y$, and $(x \wedge y)^{2}=(x y)^{2}=x^{2} y^{2}=x y=x \wedge y$ (we have used repeatedly part (a)).

Lemma 21. In any n-potent subdirectly irreducible GBL-algebra, e is join irreducible. 
Proof. Let $m<e$ be an element of the minimum nontrivial filter. If $x<e$ and $y<e$, then $x^{n} \leq m$ and $y^{n} \leq m$, as the filter generated by $x$ and the filter generated by $y$ contain $m$. Now by the identity $x(y \vee z)=x y \vee x z,(x \vee y)^{2 n}$ is the join of products of $2 n$ factors, of which $k \leq 2 n$ are equal to $x$ and $2 n-k$ are equal to $y$. Thus each product has either $\geq n$ factors equal to $x$ or $\geq n$ factors equal to $y$. Using integrality we see that each product is either $\leq x^{n}$ or $\leq y^{n}$. Thus $(x \vee y)^{2 n} \leq x^{n} \vee y^{n} \leq m<e$, therefore $x \vee y<e$.

We note that the previous result can also be deduced from Lemma 11 in [GOR], stated below in a slightly rephrased form.

Proposition 22. [GOR] A weakly commutative GBL-algebra is finitely subdirectly irreducible if and only if $e$ is join irreducible.

Lemma 23. Every subdirectly irreducible n-potent GMV-algebra is a finite chain, hence it is commutative.

Proof. Let $\mathbf{A}$ be a subdirectly irreducible $n$-potent GMV-algebra. First of all we observe that $\mathbf{A}$ is totally ordered. Indeed, any integral GMV-algebra satisfies $x \backslash y \vee$ $y \backslash x=x / y \vee y / x=e$, cf [GT05], and since by the preceding lemma $e$ is joinirreducible in $\mathbf{A}$, the claim follows.

We proceed with the proof. Let $1<k \leq n$ be the maximum natural number for which there exists $x \in A$ such that $x^{k}<x^{k-1}$, and let $x$ be such an element. Note that $x^{k}$ is an idempotent.

Claim (a): $x^{k}$ is the minimum of A. Indeed if $y<x^{k}$, then $x^{k} y=x^{k} \wedge y=y$, and using the fact that $x^{k}$ is an idempotent and Lemma 20, $y=x^{k}\left(x^{k} \backslash y\right)=x^{k} \wedge\left(x^{k} \backslash y\right)$, therefore $x^{k} \backslash y=y$. But then $e=y /\left(x^{k} \backslash y\right)=x^{k} \vee y=x^{k}$, which is impossible.

Claim (b): $x$ is a coatom. Suppose, by way of contradiction, $e>y>x$. Then $x=y(y \backslash x)$, and $x^{k}<x^{k-1}=(y(y \backslash x))^{k-1}$. Let $z=\max \{y, y \backslash x\}$. Note that $z<e$, as $x<y<e$. Then $x^{k-1} \leq z^{k-1} z^{k-1}=z^{2 k-2}$. But $z^{2 k-2} \leq z^{k}$, and repeating the argument used in the proof of Claim (a), with $x$ replaced by $z$, we see that $z^{k}$ is the minimum of A. Since $z^{k} \geq x^{k-1}>x^{k}$, a contradiction has been reached. Thus $x$ is a coatom.

Since $\mathbf{A}$ is totally ordered, there is a unique coatom $x$. Now we prove that A consists of $e$ plus all powers $x^{i}: i=1, \ldots, k$. Suppose by contradiction that for some $y \in A$ and for some $i<k, x^{i+1}<y<x^{i}$. Then $x^{i} \backslash y>x$, because $x^{i} x=x^{i+1}<y$ and $x^{i}\left(x^{i} \backslash y\right)=y$. But $x^{i} \backslash y>x$ implies $x^{i} \backslash y=e$, as $x$ is a coatom. Thus $x^{i} \leq y$, which is a contradiction.

Thus $\mathbf{A}$ is a finite chain which is generated as a monoid by its coatom, therefore it is also commutative.

As noted in Example 16, there exist noncommutative integral normal GBLalgebras. The following result shows that these examples cannot be $n$-potent.

Theorem 24. Any n-potent GBL-algebra $\mathbf{A}$ is commutative. 
Proof. Proceeding by contradiction, let $a, b \in A$ such that $a b \neq b a$. Let $U$ be maximal among all filters $F$ such that $c=((a b) \backslash(b a)) \wedge((b a) \backslash(a b)) \notin F$, and let $\mathbf{B}=\mathbf{A} / U$. Then $\mathbf{B}$ is subdirectly irreducible, and $m=c / U$ is a generator of the minimum filter $M$. Moreover, by Theorem 19, B decomposes as an ordinal sum $\mathbf{B}_{0} \oplus \mathbf{W}$, where $\mathbf{W}$ is a nontrivial subdirectly irreducible GMV-algebra. By Lemma $23, \mathbf{W}$ is a finite and commutative GMV-chain. Now let $x=a / U$ and $y=b / U$. Note that $x y \neq y x$, because $m=((x y) \backslash(y x)) \wedge((y x) \backslash(x y))<e$. Thus either $(x y) \backslash(y x)<e$ or $(y x) \backslash(x y)<e$. Suppose without loss of generality $(x y) \backslash(y x)<e$. Distinguish the following cases:

(a) $x, y \in W$. Then $x y=y x$, because $\mathbf{W}$ is commutative, and a contradiction is reached.

(b) $x \in W$ and $y \notin \mathbf{W}$, or vice versa. Then $x y=y x=x \wedge y$, and again we reach a contradiction.

(c) $x, y \in B_{0} \backslash\{e\}$. Then $x y, y x \in B_{0} \backslash\{e\}$ and $x y \backslash y x \in B_{0} \backslash\{e\}$, since $B_{0}$ is closed under residuation. On the other hand, $x y \backslash y x \in W$, because $m=$ $(x y \backslash y x) \wedge(y x \backslash x y) \leq x y \backslash y x$ and $m \in W$. Once again, a contradiction has been reached, and the proof is complete.

\section{Decision problems for GBL-algebras}

Since all finite GBL-algebras are commutative and integral, neither GBL-algebras nor integral GBL-algebras have the finite model property. In this subsection we prove that commutative and integral GBL-algebras have the finite embeddability property (FEP for short). Recall that a variety $\mathcal{V}$ has the FEP iff every finite partial subalgebra of any algebra of $\mathcal{V}$ partially embeds into a finite algebra of $\mathcal{V}$. Note that the FEP is stronger than the finite model property: for a finitely axiomatized variety $\mathcal{V}$, the finite model property implies the decidability of the equational theory of $\mathcal{V}$, while the FEP implies the decidability of the universal theory of $\mathcal{V}$.

At the end of this section we show that, without the assumption of commutativity, the quasiequational theory of all GBL-algebras is undecidable.

We start from the following lemma.

Lemma 25. Suppose $\mathbf{H}=\mathbf{H}_{0} \oplus \mathbf{W}$ is an ordinal sum of type (b) and $m$ is the minimum of $\mathbf{W}$. Then

(i) $\mathbf{H}_{0} \cup\{m\}$ is the domain of a subalgebra of $\mathbf{H}$, and

(ii) if $\mathbf{K}_{0}$ is a subalgebra of $\mathbf{H}_{0}$ and $e$ is join irreducible in $\mathbf{K}_{0}$, then $\mathbf{K}_{0}$ is a subalgebra of $\mathbf{H}$.

Proof. (i). First note that $m$ is an idempotent. Indeed, $m^{2} \leq m$, but $m^{2} \in W$, as $\mathbf{W}$ is a subalgebra of $\mathbf{H}$. Thus $m^{2}=m$, as $m$ is the minimum of $\mathbf{W}$.

Now we prove that $\mathbf{H}_{0} \cup\{m\}$ is closed under all operations of residuated lattices. It is clear that $e \in H_{0} \cup\{m\}$ and that $H_{0} \cup\{m\}$ is closed under · and under $\wedge$, since $m e=m$ and $m x=x$ for all $x \in\left(H_{0} \backslash\{e\}\right) \cup\{m\}$. Next, observe that for all $x \in H_{0} \backslash\{e\}, m \rightarrow x=x$ and $x \rightarrow m=e$ (this follows from the definition of ordinal 
sum of type (b)). Moreover, $e \rightarrow m=m$ and $m \rightarrow e=e$. Thus $\mathbf{H}_{0} \cup\{m\}$ is closed under $\rightarrow$. Finally, the join of two elements of $\mathbf{H}_{0} \backslash\{e\}$ is either in $\mathbf{H}_{0} \backslash\{e\}$ or equal to $m$. Moreover $m \vee e=e$, and for $x \in H_{0} \backslash\{e\}, m \vee x=m$. Thus $H_{0} \cup\{m\}$ is closed under $\vee$. This ends the proof of (i).

(ii). It follows from the definition of ordinal sum of type (b) that the only cases where the join in $\mathbf{H}_{0}$ of two elements $a, b$ of $\mathbf{H}_{0}$ differs from their join in $\mathbf{H}$ occurs when in $\mathbf{H}_{0} a \vee b=e$ and $a, b<e$. But this is excluded if $a, b \in K_{0}$ and $e$ is join irreducible in $\mathbf{K}_{0}$.

Theorem 26. The variety of commutative and integral GBL-algebras has the FEP.

Proof. We prove by induction on the cardinality $n$ of $\mathbf{P}$ that if $\mathbf{P}$ is a finite partial subalgebra of a commutative and integral GBL-algebra $\mathbf{A}$ such that $e \in P$, then $\mathbf{P}$ partially embeds into a finite GBL-algebra. Without loss of generality, we may assume that $\mathbf{A}$ is generated by $P$. Moreover, we may assume that $\mathbf{A}$ is subdirectly irreducible: this can be seen by an argument used in [BF00] Lemma 3.7: for $p \neq q \in$ $P$, let $\theta_{p, q}$ be maximal among the congruences which separate $p$ and $q$. Then $\mathbf{A} / \theta_{p, q}$ is subdirectly irreducible, and $\mathbf{P}$ partially embeds into $\prod_{p \neq q \in P} \mathbf{A} / \theta_{p, q}$. Assuming that the claim holds for subdirectly irreducible algebras, we have that for all $p \neq$ $q \in P, \mathbf{P} / \theta_{p, q}$ partially embeds into a finite algebra $\mathbf{B}_{p, q}$. But then $\mathbf{P}$ partially embeds into $\prod_{p \neq q \in P} \mathbf{B}_{p, q}$, which is a finite algebra.

Thus we can suppose that $\mathbf{A}$ is subdirectly irreducible. Moreover the above argument shows that we may assume without loss of generality that any nontrivial congruence $\theta$ of $\mathbf{A}$ identifies two distinct elements of $\mathbf{P}$. Thus, we assume that $\mathbf{P}$ has cardinality $n$, and that the claim holds for all finite partial subalgebras of cardinality $<n$ of any commutative and integral GBL-algebra. Since $\mathbf{A}$ is subdirectly irreducible, by Proposition 22, $e$ is join irreducible in $\mathbf{A}$, hence it is join irreducible in $\mathbf{P}$. Now decompose $\mathbf{A}$ as $\mathbf{H} \oplus \mathbf{W}$, where $\mathbf{H}$ is a commutative and integral GBL-algebra and $\mathbf{W}$ is a subdirectly irreducible nontrivial commutative and integral GMV-algebra. Note that $\mathbf{H}$ is closed under the operations $\cdot \wedge$ and $\rightarrow$ of $\mathbf{A}=\mathbf{H} \oplus \mathbf{W}$. Moreover, the join in $\mathbf{A}$ of two elements of $\mathbf{H} \backslash\{e\}$ is either in $\mathbf{H} \backslash\{e\}$ or the minimum $m$ of $\mathbf{W}$. Now distinguish three cases:

Case (a): $e$ is join irreducible in $\mathbf{H}$. Then since Wajsberg hoops have the FEP [BF00], commutative and integral GMV-algebras also have the FEP, being term equivalent to Wajsberg hoops. Thus $\mathbf{W} \cap \mathbf{P}$ partially embeds into a finite GMValgebra, $\mathbf{V}$ say. Moreover, since $\mathbf{A}$ is generated by $\mathbf{P}$ and $\mathbf{H}$ is a subalgebra of $\mathbf{A}$, at least one element of $\mathbf{P}$ is in $\mathbf{W} \backslash\{e\}$, therefore $\mathbf{H} \cap \mathbf{P}$ has cardinality $<n$, and by the induction hypothesis it partially embeds into a finite GBL-algebra $\mathbf{K}$. But then $\mathbf{P}$ partially embeds into $\mathbf{K} \oplus \mathbf{V}$ (the ordinal sum is of type (a) or of type (b) according to whether $e$ is join irreducible in $\mathbf{K}$ or not).

Case (b): $e$ is not join irreducible in $\mathbf{H}$ and $\mathbf{W}$ has more than two elements. Then since $\mathbf{H} \cup\{m\}$ is a subalgebra of $\mathbf{A}$ (Lemma 25) and $\mathbf{A}$ is generated by $\mathbf{P}$, $\mathbf{W} \cap \mathbf{P}$ must contain some element which is not in the subalgebra of $\mathbf{A}$ generated by $\mathbf{H}$. Therefore, $\mathbf{H} \cap \mathbf{P}$ has cardinality $<n$. Thus we can proceed as in case (a). 
Case (c): $e$ is not join irreducible in $\mathbf{H}$ and $\mathbf{W}$ has only two elements. Then $\mathbf{W}$ has a minimum $m$, which is necessarily an idempotent. Now note that $\mathbf{W}$ is a nontrivial filter of $\mathbf{A}$, therefore by our assumption on $\mathbf{A}$, the congruence $\theta$ associated to $\mathbf{W}$ identifies at least two different elements of $\mathbf{P}$. It follows that $\mathbf{P} / \theta$ is a partial subalgebra of $\mathbf{A} / \theta$ of cardinality $<n$, therefore by the induction hypothesis it partially embeds into a finite GBL-algebra $\mathbf{K}$. Note that $\theta$ does not identify different elements of $\mathbf{H} \backslash\{e\}$ : indeed, if $(x, y) \in \theta$, then $m \leq x \rightarrow y$ and $m \leq y \rightarrow x$, therefore $m x \leq y$ and $m y \leq x$. But if $x \in H \backslash\{e\}$, then $m x=x$, therefore $x \leq y$. Similarly $y \leq x$, hence $y=x$. Moreover, $\theta$ does not identify $e$ with elements of $\mathbf{H} \backslash\{e\}$, because the equivalence class of $e$ is $\mathbf{W}$. For the same reason, $\theta$ does not identify $m$ with any element of $\mathbf{H} \backslash\{e\}$. Hence the only elements of $\mathbf{A}$ identified by $\theta$ are $m$ and $e$, therefore $m$ and $e$ must be in $\mathbf{P}$. Thus $\mathbf{P}$ partially embeds into the finite algebra $\mathbf{K} \oplus \mathbf{W}$.

\section{Corollary 27.}

(a) The universal theory of commutative and integral GBL-algebras is decidable.

(b) The universal theory of commutative GBL-algebras is decidable.

Proof. (a) This follows from Theorem 26 because the class is finitely axiomatized.

(b) By Proposition 4, any commutative GBL-algebra decomposes as a direct product of a commutative and integral GBL-algebra and an abelian $\ell$-group. Thus any subdirectly irreducible commutative GBL-algebra is either an abelian $\ell$-group or a commutative and integral GBL-algebra. It follows that a quasiequation holds in all commutative GBL-algebras if and only if it holds in all abelian $\ell$-groups and in all commutative and integral GBL-algebras. So the decidability of the quasiequational theory of commutative GBL-algebras follows from (a) and from the decidability of the quasiequational theory of abelian $\ell$-groups (cf [We86]).

Since commutative GBL-algebras are closed under products, the universal theory this class is also decidable by McKinsey's lemma (see e.g. [Ho93] Cor. 9.1.7).

Whilst the quasiequational theory of commutative GBL-algebras is decidable, the quasiequational theory of GBL-algebras is undecidable, as shown by the next proposition.

Recall that an $\ell$-group can be regarded as a residuated lattice, with $x \backslash y=x^{-1} y$ and $y / x=y x^{-1}$, and that the inverse operation can be written in the language of residuated lattices as $x^{-1}=x \backslash e$.

Proposition 28. To each quasiequation $\Phi$ of residuated lattices we can constructively associate a quasiequation $\Phi^{\prime}$ such that $\Phi$ holds in all $\ell$-groups iff $\Phi^{\prime}$ holds in all GBL-algebras. Thus the quasiequational theory of GBL-algebras is undecidable.

Proof. Let $\Phi$ be $\left(\phi_{1} \& \ldots \& \phi_{n}\right) \Rightarrow \psi$, where $\phi_{1}, \ldots, \phi_{n}, \psi$ are equations in the language of residuated lattices. Let $\phi=\left(\phi_{1} \& \ldots \& \phi_{n}\right)$, and let $x_{1}, \ldots, x_{k}$ be the variables occurring in $\Phi$. For $i=1, \ldots, k$, let $\gamma_{i}$ denote the equation $x_{i}\left(x_{i} \backslash e\right)=e$. Let $\gamma=\gamma_{1} \& \ldots \& \gamma_{k}$, and let $\Phi^{\prime}=(\gamma \& \phi) \Rightarrow \psi$. We claim that $\Phi$ holds in all $\ell$-groups 
iff $\Phi^{\prime}$ holds in all GBL-algebras. By [GG83] the word problems for $\ell$-groups, and hence the quasiequational theory, are undecidable, so the claim will imply that the quasiequational theory of GBL-algebras is also undecidable.

If $\Phi^{\prime}$ holds in all GBL-algebras, then it holds in all $\ell$-groups. But $\gamma_{1}, \ldots, \gamma_{k}$ are valid in all $\ell$-groups, therefore in $\ell$-groups $\Phi^{\prime}$ is equivalent to $\Phi$. It follows that $\Phi$ holds in all $\ell$-groups.

Conversely, assume that $\Phi$ holds in all $\ell$-groups. Let $\mathbf{A}$ be any GBL-algebra. By Proposition 4 we can write it as $\mathbf{A}=\mathbf{B} \times \mathbf{G}$, where $\mathbf{B}$ is an integral GBL-algebra and $\mathbf{G}$ is an $\ell$-group. Let $v$ be a valuation of variables into $\mathbf{A}$ such that $\gamma \& \phi$ is true under $v$. We can write $v\left(x_{i}\right)$ as $v\left(x_{i}\right)=\left(b_{i}, g_{i}\right)$ with $b_{i} \in B$ and $g_{i} \in G$. Then by the truth of $\gamma_{i}$ under the valuation $v$, in $\mathbf{B}$ we must have $b_{i}\left(b_{i} \backslash e\right)=e$, which in turn implies $b_{i}=e$, as $\mathbf{B}$ is integral. Thus the subalgebra $\mathbf{C}$ of $\mathbf{A}$ generated by $v\left(x_{1}\right), \ldots, v\left(x_{n}\right)$ is in fact a subalgebra of $\{e\} \times \mathbf{G}$, therefore it is an $\boldsymbol{\ell}$-group. Hence $\Phi$ is valid in $\mathbf{C}$, and therefore it is true in $\mathbf{A}$ under the valuation $v$. Finally, the truth of $\phi$ under $v$ implies that $\psi$ is also true under $v$. Thus $\Phi^{\prime}$ is valid in $\mathbf{A}$, and the claim is proved.

\section{Poset sum representability for finite GBL-algebras}

In this section we define a generalization of ordinal sums, called poset sums. Using the Blok-Ferreirim theorem for normal GBL-algebras, we proceed to show that every finite GBL-algebra is isomorphic to a poset sum of finite Wajsberg chains.

It is proved in [JM06] that any finite GBL-algebra $\mathbf{A}$ is commutative, and that the set $I(\mathbf{A})$ of idempotents forms a subalgebra that satisfies $x y=x \wedge y$ and hence is a Brouwerian algebra. Let $P$ be the poset of join-irreducibles of this Brouwerian algebra. Each $i \in P$ has a unique lower cover $i^{*} \in I(\mathbf{A})$. We show, with the help of Theorem 19, that the interval $\mathbf{A}_{i}=\left[i^{*}, i\right]$ is a chain, and has the multiplicative structure of a Wajsberg hoop. The GBL-algebra $\mathbf{A}$ can be reconstructed from a subset of the direct product of these Wajsberg chains.

For BL-algebras, this structure result is proved in [DL03]. In this case the representability of BL-algebras implies that the poset of join-irreducibles is a forest (i.e. the downset of any element is a chain). The approach taken here is somewhat simpler and shows that representability plays no role in this result.

A generalized ordinal sum construction for bounded integral residuated lattices is defined as follows:

Let $P$ be a poset, and let $\mathbf{A}_{i}(i \in P)$ be a family of residuated lattices. In addition we require that for nonmaximal $i \in P$ each $\mathbf{A}_{i}$ is integral, and for nonminimal $i \in P$ each $\mathbf{A}_{i}$ has a least element denoted by $0_{i}$. The poset sum is defined as

$$
\bigoplus_{i \in P} A_{i}=\left\{a \in \prod_{i \in P} A_{i}: a_{j}<e \Longrightarrow a_{k}=0_{k} \text { for all } j<k\right\} .
$$

This subset of the product contains the constant function $\underline{e}$. Note that an element $a$ is in the poset sum if and only if $\left\{i \in P: 0_{i}<a_{i}<\bar{e}\right\}$ is an antichain and 
$\left\{i \in P: a_{i}=e\right\}$ is downward closed (hence $\left\{i \in P: a_{i}=0_{i}\right\}$ is upward closed). The operations $\wedge, \vee$ and $\cdot$ are defined pointwise (as in the product). For the definition of the residuals, we have

$$
\begin{aligned}
& (a \backslash b)_{i}= \begin{cases}a_{i} \backslash b_{i} & \text { if } a_{j} \leq b_{j} \text { for all } j<i \\
0_{i} & \text { otherwise. }\end{cases} \\
& (a / b)_{i}= \begin{cases}a_{i} / b_{i} & \text { if } b_{j} \leq a_{j} \text { for all } j<i \\
0_{i} & \text { otherwise. }\end{cases}
\end{aligned}
$$

If $i$ is a maximal (minimal) element of $P$, we refer to $\mathbf{A}_{i}$ as a maximal (minimal) summand.

Theorem 29. The poset sum of residuated lattices is again a residuated lattice. If all maximal summands are integral then the poset sum is integral, and if all minimal summands have a least element, then the poset sum has a least element.

Proof. Let $P$ be a poset, and $\mathbf{B}=\bigoplus_{i \in P} \mathbf{A}_{i}$ a poset sum of residuated lattices. It is straightforward to check that $B$ is a submonoid and sublattice of $\prod_{i \in P} \mathbf{A}_{i}$. To show that $\mathbf{B}$ has a residual defined as above, we need to prove that (i) for all $a, b \in B, a \backslash b \in B$ and (ii) for all $x \in B, a x \leq b$ if and only if $x \leq a \backslash b$.

For (i), let $j<k$ and assume $(a \backslash b)_{j}<e$. If $a_{l} \leq b_{l}$ for all $l<k$, then in particular $a_{j} \leq b_{j}$, and $a_{l} \leq b_{l}$ for all $l<j$. Hence $(a \backslash b)_{j}=a_{j} \backslash b_{j}=e$, contradicting the assumption $(a \backslash b)_{j}<e$. So we conclude $a_{l} \not \leq b_{l}$ for some $l<k$, and thus $(a \backslash b)_{k}=0_{k}$ as required.

For (ii), note that $a_{i} x_{i} \leq b_{i}$ for all $i \in P$ iff $x_{i} \leq a_{i} \backslash b_{i}$ (in $\mathbf{A}_{i}$ ) for all $i \in P$. Assume that $a_{i} x_{i} \leq b_{i}$ holds for all $i \in P$. If $a_{l} \not \leq b_{l}$ for some $l<i$, then $x_{l} \leq a_{l} \backslash b_{l}<e$, hence $x_{i}=0_{i}$. It follows that for all $i \in P, x_{i} \leq(a \backslash b)_{i}$. The reverse implication is obvious since $(a \backslash b)_{i} \leq a_{i} \backslash b_{i}$.

Poset sums (as defined here) already require that all nonmaximal summands are integral, so if all maximal summands are integral as well then the identity $\underline{e}$ of the poset sum is the top element. Similarly it is easy to see that if all minimal summands have a bottom element then so does the poset sum.

We note that poset sums generalize both ordinal sums and direct products. Indeed, if the poset $P$ is a chain, the poset sum produces an ordinal sum (of type (a) or (b) since we are assuming that $\mathbf{A}_{i}$ has a least element for all nonminimal $i \in P$ ), and if $P$ is an antichain then it produces a direct product. Since poset sums are generalizations of ordinal sums, we cannot expect the varieties of Boolean algebras, MV-algebras or involutive lattices to be closed under poset sums. However it does preserve the defining property of generalized basic logic.

Theorem 30. The variety of integral GBL-algebras is closed under poset sums.

Proof. Suppose the factors $\mathbf{A}_{i}$ of the poset sum B all satisfy the GBL-quasi-identity $x \leq y \Rightarrow x=(x / y) y=y(y \backslash x)$, let $a \leq b \in B$ and consider $i \in P$. If $b_{l} \not \leq a_{l}$ for some $l<i$ then $(b \backslash a)_{i}=0_{i}$ and $a_{l}<b_{l} \leq e$, whence $a_{i}=0_{i}=b_{i}(b \backslash a)_{i}$. Here we made 
use of the fact that $a$ is a member of the poset sum, and that $0 x=0=x 0$ holds in any bounded residuated lattice. On the other hand, if $b_{l} \leq a_{l}$ for all $l<i$ then $a_{i}=b_{i}\left(b_{i} \backslash a_{i}\right)=b_{i}(b \backslash a)_{i}$. Thus $a=b(b \backslash a)$, and $a=(a / b) b$ is proved similarly.

In fact, for GBL-algebras, this construction describes all the finite members. For a residuated lattice $\mathbf{A}$ and an idempotent element $i$ of $A$, we let $\downarrow i=\{a \in A: a \leq i\}$ and $\mathbf{A}_{\downarrow i}=\left(\downarrow i, \cdot, \backslash_{i}, / i, \vee, \wedge, i\right)$, where $a \backslash_{i} b=(a \backslash b) \wedge i$ and $a /{ }_{i} b=(a / b) \wedge i$.

Lemma 31. For any integral GBL-algebra $\mathbf{A}$ and any idempotent element $i$ of $\mathbf{A}$, the map $\hat{i}: A \rightarrow \downarrow i$ given by $\hat{i}(a)=a \wedge i$ is a homomorphism from $\mathbf{A}$ to $\mathbf{A}_{\downarrow}$. Hence the algebra $\mathbf{A}_{\downarrow i}$ is an integral GBL-algebra.

Proof. By Lemma 20(a) for any $x \in A$ we have $x \wedge i=i x=x i$ hence $\hat{i}(a b)=a b i^{2}=$ $a i b i=\hat{i}(a) \hat{i}(b)$. Clearly $\hat{i}(a \wedge b)=\hat{i}(a) \wedge \hat{i}(b)$, and $\hat{i}(a \vee b)=\hat{i}(a) \vee \hat{i}(b)$ follows by distributivity. Finally $\hat{i}(a) \backslash{ }_{i} \hat{i}(b)=((a \wedge i) \backslash(b \wedge i)) \wedge i=((a i) \backslash b) \wedge((a i) \backslash i) \wedge i=$ $(i \backslash(a \backslash b)) \wedge i=i(i \backslash(a \backslash b))=(a \backslash b) \wedge i=\hat{i}(a \backslash b)$. The argument for $/$ is similar.

The next result follows from the observation that lattice operations and residuals are first-order definable from the partial order and the monoid operation of a residuated lattice.

Lemma 32. Suppose $\mathbf{A}, \mathbf{B}$ are residuated lattices and $h: A \rightarrow B$ is an orderpreserving and order-reflecting monoid isomorphism. Then $h$ is a residuated lattice isomorphism.

Theorem 33. Let $\mathbf{A}$ be a finite GBL-algebra and let $P$ be the set of all joinirreducible idempotents of $\mathbf{A}$. For $i \in P$, let $i^{*} \in A$ be the unique maximal idempotent below $i$, and let $A_{i}=\left\{x: i^{*} \leq x \leq i\right\}=\left[i^{*}, i\right]$. Then $\mathbf{A}_{i}=\left(A_{i}, \wedge, \vee, \cdot, \backslash^{i}, /{ }^{i}, i\right)$ is a Wajsberg chain and $\mathbf{A} \cong \bigoplus_{i \in P} \mathbf{A}_{i}$.

Proof. By [JT02], the congruence lattice of a finite residuated lattice is dually isomorphic to the poset of negative central idempotents, hence if the identity in a finite residuated lattice is join irreducible then this algebra is subdirectly irreducible.

Thus for a join irreducible idempotent $i$, the algebra $\mathbf{A}_{\downarrow i}$ is a subdirectly irreducible GBL-algebra. Note that the bottom element of $\mathbf{A}$ is not join-irreducible (since it is the value of the empty join). It follows from Theorem 19 that this algebra decomposes into the ordinal sum $\mathbf{B} \oplus \mathbf{W}$ where $\mathbf{W}$ is a nontrivial subdirectly irreducible GMV-algebra. Since it is finite, $\mathbf{W}$ is commutative and therefore a Wajsberg chain. The least element of $\mathbf{W}$ is the unique maximal idempotent $i^{*}$, hence $\mathbf{W}$ is $\mathbf{A}_{i}$ as defined in the statement of the theorem.

Define $h_{i}: A \rightarrow A_{i}$ by $h_{i}(a)=(a \wedge i) \vee i^{*}$ and let $h: A \rightarrow \prod_{i \in P} A_{i}$ be given by $h(a)=\left(h_{i}(a): i \in P\right)$.

Next we prove that $h$ maps into the poset sum of the $A_{i}$. Suppose $i<j$ and $h_{j}(a) \neq j^{*}$. We need to show that $h_{i}(a)=i$. Since $h_{j}(a)=(a \wedge j) \vee j^{*}$, it follows that $a \wedge j \not \leq j^{*}$. Since $\mathbf{A}_{\downarrow j}$ decomposes as an ordinal sum with the chain $A_{j}$ as upper component, we have $a \wedge j>j^{*}$. Thus $a>j^{*} \geq i$, which implies $h_{i}(a)=i$. 
GBL-algebras are distributive, hence $h$ is a lattice homomorphism. By Lemma 31, $(a b) \wedge i=(a \wedge i)(b \wedge i)$. Also, for any idempotent $j$ we have $(a \vee j)(b \vee j)=$ $a b \vee a j \vee b j \vee j^{2}=a b \vee j$ so $h_{i}(a b)=h_{i}(a) h_{i}(b)$.

By Lemma 32 it suffices to prove that $h$ is a bijection, then it follows that $h$ preserves the residuals. Define $g: \bigoplus_{i \in P} A_{i} \rightarrow A$ by $g(x)=\bigvee\left\{x_{i}: x_{k}=\right.$ $k$ for all $k<i\}$. We claim that $g$ is the inverse of $h$. Note that $g(h(a))=\bigvee\{(a \wedge$ i) $\vee i^{*}:(a \wedge k)=k$ for all $\left.k<i\right\}$. Since $i^{*}=\bigvee^{\mathbf{A}}\{k: k<i\}$, we have $g(h(a))=$ $\bigvee\left\{(a \wedge i): i^{*} \leq a\right\}$.

Since the set of idempotents of $\mathbf{A}$ is closed under meets, for any join irreducible $p \in A$ there exists a smallest idempotent $i \geq p$.

Claim: $i$ is join irreducible. If $p$ is idempotent, then $i=p$ and the claim holds. So suppose $p^{2}<p$. If $i$ is not join irreducible then $i$ has at least two lower covers, say $b, c$. If $p \not \leq b$ then $p b \leq p \wedge b<p$ and $p^{2} \vee p b=p(p \vee b)=p i=p \wedge i=p$ contradicting the assumption that $p$ is join irreducible. If $p$ is below both $b, c$, let $\bar{b}, \bar{c}$ be the maximal idempotents below $b, c$ respectively. By choice of $i$, we have $p \not \leq \bar{b}, \bar{c}$, and by Lemma 2 of [JM06], $\bar{b}, \bar{c}$ are not below $p$ and $\bar{b} \vee \bar{c}=i$. Hence $(p \wedge \bar{b}) \vee(p \wedge \bar{c})=p$, again contradicting the join irreducibility of $p$. This proves the claim.

It follows that each join irreducible $p \leq a$ of $\mathbf{A}$ lies in a unique interval $\left[i^{*}, i\right]$, hence $p=a \wedge i$ for some $i^{*} \leq a$. Thus $g(h(a))=a$.

It remains to show that for all $x$ in the poset sum $h(g(x))=x$. By definition $h(g(x))=(g(x) \wedge i) \vee i^{*}=\left(\bigvee\left\{x_{j}: x_{k}=k\right.\right.$ for all $\left.\left.k<j\right\} \wedge i\right) \vee i^{*} \in\left[i^{*}, i\right]$. Using distributivity we may write this in the form $\bigvee\left\{\left(x_{j} \wedge i\right) \vee i^{*}: x_{k}=k\right.$ for all $\left.k<j\right\}$.

Now suppose $x_{i}=i$. Then $x_{k}=k$ for all $k<i$, so $x_{i}$ is one of the joinands, hence $h_{i}(g(x))=i=x_{i}$. On the other hand, if $x_{i}<i$ then $h_{i}(g(x)) \geq x_{i}$ (even in case $x_{k}<k$ for some $k<i$, since then $\left.x_{i}=i^{*}\right)$. We need to show that $x_{j} \wedge i \leq x_{i}$ for all other joinands, i.e. whenever $x_{k}=k$ for all $k<j$. In this case we know $j \ngtr i$ since $x_{i}<i$. If $j<i$ then $x_{j} \leq j \leq i^{*} \leq x_{i}$, and if $i, j$ are incomparable, then $i \wedge j$ is an idempotent $\leq i^{*}$ so $x_{j} \wedge i \leq j \wedge i \leq i^{*} \leq x^{i}$.

Moreover, there is a bijective correspondence between finite GBL-algebras and finite posets labeled with natural numbers $>1$, denoting the size of the corresponding Wajsberg chain in the poset sum.

If the poset is a forest, the GBL-algebra is representable hence, adding a least element 0 to the type, it becomes a BL-algebra. Thus the result proved here generalizes the one in [DL03].

Our representation result is useful for constructing and counting finite GBLalgebras. For example, Figure 1 shows the lattice structure of a GBL-algebra with 17 elements that is obtained from a poset sum of a 2, 3, 4, and 5-element Wajsberg chain over the poset $\mathbf{2} \times \mathbf{2}$ (the join irreducible idempotents are denoted by black dots). Theorem 33 implies that the same lattice supports $2^{6}=64$ nonisomorphic GBL-algebras since there are six other join irreducibles that could be chosen as idempotents. 

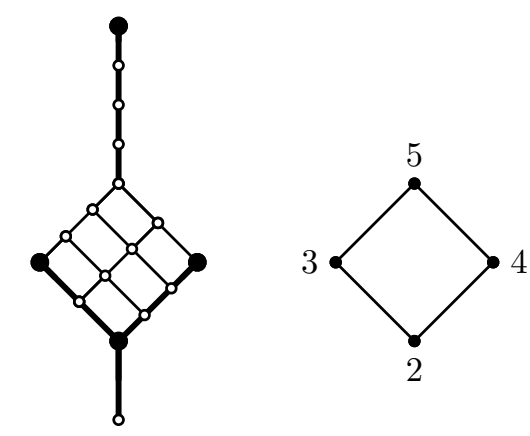

Figure 1. A GBL-algebra and its labeled poset of join-irreducibles

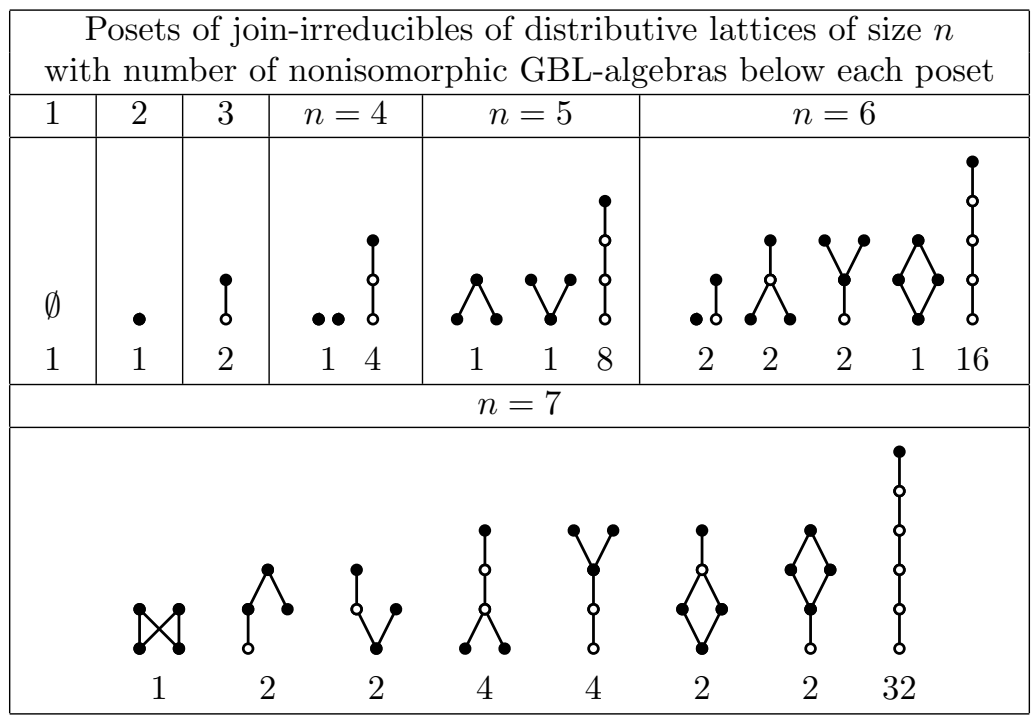

\begin{tabular}{|l|ccccccc|}
\hline Size $n=$ & 1 & 2 & 3 & 4 & 5 & 6 & 7 \\
\hline GBL-algebras & 1 & 1 & 2 & 5 & 10 & 23 & 49 \\
si GBL-algebras & 0 & 1 & 2 & 4 & 9 & 19 & 42 \\
BL-algebras & 1 & 1 & 2 & 5 & 9 & 20 & 38 \\
si BL-algebras & 0 & 1 & 2 & 4 & 8 & 16 & 32 \\
\hline
\end{tabular}

Figure 2. Counting nonisomorphic GBL-algebras of size $n$

When counting the number of nonisomorphic GBL-algebras of size $n$, it is convenient to consider the poset of all join irreducibles of the lattice reduct. Elements that have no upper cover, more than one upper cover or whose upper cover has more than one lower cover must be idempotents of the GBL-algebra (denoted by 
black dots in Figure 2). The remaining elements each have unique upper covers of which they are the only lower cover. These elements can be chosen as idempotents (or not), giving rise to distinct GBL-algebras (see Figure 2). A finite GBL-algebra is subdirectly irreducible iff the poset of join-irreducibles has a top element, and the algebra is representable (and hence expands to a finite BL-algebra) iff the poset of join-irreducibles is a forest. Hence the lower table of Figure 2 is obtained by inspecting the posets in the upper table. In particular, since subdirectly irreducible BL-algebras are chains, it follows that for $n>1$ there are precisely $2^{n-2}$ nonisomorphic subdirectly irreducible $n$-element BL-algebras.

Note that the variety of idempotent GBL-algebras is (term-equivalent to) the variety of Brouwerian algebras. Now the finite members in this variety are just finite distributive lattices, expanded with the residual of the meet operation. Thus Birkhoff's duality between finite posets and finite distributive lattices shows that finite Brouwerian algebras are isomorphic to poset sums of the two-element generalized Boolean algebra (= two-element Wajsberg chain). Using the preceding theorem, this result can be generalized to $n$-potent GBL-algebras.

Corollary 34. Any finite n-potent GBL-algebra is isomorphic to a poset sum of Wajsberg chains with at most $n$ elements.

We would like to thank James Raftery and an anonymous referee for many valuable comments that have substantially improved this paper.

\section{REFERENCES}

[AM03] P. Aglianò, F. Montagna, Varieties of BL-algebras I: general properties, Journal of Pure and Applied Algebra 181, (2003), 105-129.

[AF88] M. Anderson, T. Feil, "Lattice Ordered Groups, An Introduction", D. Reidel Publishing Company, Dordrecht, Boston, Lancaster, Tokyo 1988.

[BF00] W. J. Blok, I. M. A. Ferreirim, On the structure of hoops, Algebra Universalis 43 (2000), 233-257.

[BT03] K. Blount, C. Tsinakis, The structure of residuated lattices, International Journal of Algebra and Computation 13(4), (2003), 437-461.

[Bo69] B. Bosbach, Komplementäre Halbgruppen, Axiomatik und Arithmetik, Fundamenta Mathematicae 64, (1969), 257-287.

[BO75] J. R. Büchi, T. M. Owens, Complemented monoids and hoops, 1975, unpublished manuscript.

[Bu05] M. Busaniche, Decomposition of BL chains, Algebra Universalis, 52, n. 4, (2005), 519-525.

[COM00] R. Cignoli, I. D’Ottaviano, D. Mundici, Algebraic foundations of many-valued reasoning, Kluwer Academic Publishers, Dordrecht, Boston, London 2000.

[CEGT00] R. Cignoli, F. Esteva, L. Godo, A. Torrens, Basic Fuzzy Logic is the logic of continuous t-norms and their residua, Soft Computing 4, (2000), 106-112.

[Co82] W. H. Cornish, BCK-algebras with a supremum, Math. Japon. 27 (1982), no. 1, 63-73.

[DGJ02] A. Di Nola, G. Georgescu, A. Iorgulescu, Pseudo BL-algebras I, Multiple-Valued Logic 8 (2002), no. 5-6, 673-714.

[DL03] A. Di Nola, A. Lettieri, Finite BL-algebras, Discrete Mathematics 269 (2003), no.1-3, 93-112. 
[Dv02] A. Dvurečenskij, Pseudo MV-algebras are intervals in $\ell$-groups, Journal of Australian Mathematical Society 70 (2002), 427-445.

[Dv06] A. Dvurečenskij, Aglianò-Montagna Type Decomposition of Pseudo Hoops and its Applications, Technical Report 2006.

[Fe92] I. M. A. Ferreirim, On varieties and quasivarieties of hoops and their reducts, $\mathrm{PhD}$ thesis, University of Illinois at Chicago, 1992.

[GOR] N. Galatos, J. S. Olson, J. G. Raftery, Irreducible residuated semilattices and finitely based varieties, Reports on Mathematical Logic, to appear.

[GO06] N. Galatos, H. Ono, Algebraization, parametrized local deduction theorem and interpolation for substructural logics over FL, Studia Logica 83, (2006), 1-32.

[GT05] N. Galatos, C. Tsinakis, Generalized MV-algebras, Journal of Algebra 283 (2005), $254-291$.

[GLP05] G. Georgescu, L. Leuştean, V. Preoteasa, Pseudo hoops, Journal of Multiple Valued Logic and Soft Computing 11 (2005), 153-184.

[GG83] A. M. W. Glass, Y. Gurevich, The word problem for lattice-ordered groups, Trans. Amer. Math. Soc., 280 (1983), 127-138.

[Ha98] P. Hájek, "Metamathematics of Fuzzy Logic", Trends in Logic, Studia Logica Library no. 4, Kluwer Academic Publishers, Dordrecht, Boston, London 1998.

[Ha98a] P. Hájek, Basic Fuzzy Logic and BL-algebras, Soft Computing 2 (1998), 124-128.

[Ho93] W. Hodges, "Model Theory", Encyclopedia of Mathematics and its Applications Vol. 42, Cambridge University Press, 1993.

[JM06] P. Jipsen, F. Montagna, On the structure of generalized BL-algebras, Algebra Universalis 55 (2006), 226-237.

[JT02] P. Jipsen, C. Tsinakis, A survey on residuated lattices, in: J. Martinez (Ed.), Ordered Algebraic Structures, Kluwer 2002, 19-56.

[Mu86] D. Mundici, Interpretations of $A F C^{*}$-algebras in Eukasiewicz sentential calculus, Journal of Functional Analysis 65 (1986), 15-63.

[We86] V. Weispfenning, The complexity of the word problem for abelian $\ell$-groups, Theoretical Computer Science 48 (1986), 127-132.

Chapman University, Department of Mathematics and Computer Science, Orange, CA 92866, USA

E-mail address: jipsen@chapman.edu

University of Siena, Department of Mathematics and Computer Science, Pian dei Mantellini 44, 53100 Siena, Italy

E-mail address: montagna@unisi.it 